\title{
Quartz-enhanced conductance spectroscopy for nanomechanical analysis of polymer wire
}

\author{
Huadan Zheng, ${ }^{1,2}$ Xukun Yin, ${ }^{1,2}$ Guofeng Zhang, ${ }^{1,2}$ Lei Dong, ${ }^{1,2,3, a)}$ Hongpeng Wu, ${ }^{1,2}$ \\ Xiaoli Liu, ${ }^{1,2}$ Weiguang Ma, ${ }^{1,2}$ Lei Zhang, ${ }^{1,2}$ Wangbao Yin, ${ }^{1,2}$ Liantuan Xiao, ${ }^{1,2}$ \\ Suotang Jia, ${ }^{1,2}$ and Frank K. Tittel ${ }^{3}$ \\ ${ }^{1}$ State Key Laboratory of Quantum Optics and Quantum Optics Devices, Institute of Laser Spectroscopy, \\ Shanxi University, Taiyuan 030006, China \\ ${ }^{2}$ Collaborative Innovation Center of Extreme Optics, Shanxi University, Taiyuan 030006, China \\ ${ }^{3}$ Department of Electrical and Computer Engineering, Rice University, Houston, Texas 77004, USA
}

(Received 19 September 2015; accepted 12 November 2015; published online 30 November 2015)

Quartz-enhanced conductance spectroscopy is developed as an analytical tool to investigate dynamic nanomechanical behaviors of polymer wires, in order to determine the glass transition temperature $\left(T_{\mathrm{g}}\right)$. A polymethyl methacrylate (PMMA) microwire with a diameter of $10 \mu \mathrm{m}$ was bridged across the prongs of a quartz tuning fork (QTF). With the advantage of QTF self-sensing as compared with micro-cantilevers or other resonators, the resonance frequency and $\mathrm{Q}$ factor can be directly determined by means of its electrical conductance spectra with respect to the frequency of the external excitation source ( $\mathrm{d} / \mathrm{d} V$ vs $f$ ), and therefore, no optical beam is required. The $T_{\mathrm{g}}$ of the PMMA microwire was determined by the maximum loss modulus of the QTF, calculated from the resonance frequency and the $\mathrm{Q}$ factor as a function of temperature. The measured $T_{\mathrm{g}}$ of the PMMA is $103{ }^{\circ} \mathrm{C}$ with an error of $\pm 2{ }^{\circ} \mathrm{C}$. Both heating/cooling and physical aging experiments were carried out, demonstrating that the technique is both reversible and reproducible. (c) 2015 AIP Publishing LLC. [http://dx.doi.org/10.1063/1.4936648]

The glass transition, which is the primary transition of polymer mechanical states, is the most important mechanical transition phenomenon in amorphous polymers. The glass transition temperature $\left(T_{\mathrm{g}}\right)$ is a significant property of amorphous polymers and is the temperature where the mechanical states of the polymer changes between a hard and relatively brittle state called the glass state and a molten or rubber-like state called the rubbery state. From a microcosmic viewpoint, the transition of a mechanical state is due to the "vitrifying" and "thawing" of the polymer chains. If the polymer melts are cooled below the $T_{\mathrm{g}}$ over a period that is shorter than the time required for molecular rearrangements to accommodate the temperature changes, the vitrified polymer chains are kinetically trapped in a nonequilibrium state. ${ }^{1,2}$ The glass transition has a significant impact on the polymer properties of thermodynamics, mechanics, and electrics.

The glass transition in polymers can be observed experimentally by measuring any one of several basic thermodynamic, physical, mechanical, or electrical properties as a function of temperature. ${ }^{3,4}$ Dilatometry is the established method for volume temperature measurements, in which the polymer is confined by mercury and its volume change is recorded as a function of increasing temperature. Thermal methods, differential thermal analysis (DTA), or differential scanning calorimetry (DSC) yield peaks relating to endothermic and exothermic transitions and show changes in the heat capacity during the glass transition. Mechanical methods measure the dynamic mechanical parameters of elastic modus $\left(E^{\prime}\right)$, loss modulus $\left(E^{\prime \prime}\right)$, and loss tangent $(\tan \delta)$ of the

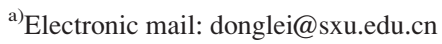

polymer when a force or deformation is exerted. The mechanical methods provide the most direct determination of the glass transition and result in a high accuracy of the $T_{\mathrm{g}}$ measurements. ${ }^{5}$ Micro-cantilevers or micromechanical string resonators were recently developed to directly investigate the interaction between the thermodynamics and mechanics. ${ }^{4,6,7}$ The analytes were prepared by inkjet printing or spray coating methods, and the deformation of cantilevers or string resonators were measured by an optical beam technique.

Quartz tuning forks (QTF) are extremely sensitive force sensors that can be used for femto-newton force sensing, ${ }^{8}$ electric field intensity distribution detecting at subwavelength resolution, ${ }^{9}$ sub-atomic resolution imaging of the charge distribution of a single atom, ${ }^{10}$ and ppb (part per billion by volume)-level trace gas detection. ${ }^{11-19}$ A nanowire modified QTF can be used to measure the ultrahigh interlayer friction in multi-walled nanotubes or the stress change in the wire upon exposure to organic vapors by measuring the resonance frequency shift. ${ }^{20-23}$ Compared with cantilevers and string resonators, ${ }^{24,25}$ the QTFs have a significant advantage of self-sensing since the resonance frequency and Q factor of a QTF can be directly read out by means of its electrical conductance spectra with respect to the frequency of the external excitation source ( $\mathrm{d} I / \mathrm{d} V$ vs $f$ ), and therefore, requires no optics. QTFs can be easily implemented if operated in ultrahigh vacuum and temperatures. ${ }^{26}$ Furthermore, QTFs have very high Q factors since quartz is one of the materials with the lowest internal mechanical losses. ${ }^{27-29}$

In this letter, quartz-enhanced conductance spectroscopy (QECS) was developed as an analytical tool to investigate dynamic nanomechanical behaviors of polymethyl methacrylate (PMMA) of a few nanograms, in order to determine the 
glass transition temperature $\left(T_{\mathrm{g}}\right)$. A PMMA microwire weighing several nanograms was bridged across the prongs of a QTF to analyze the nanomechanics of PMMA at different temperatures. The resonance frequency and $\mathrm{Q}$ factor of the microwire modified QTF (MM-QTF), which are related to the elastic modulus and loss tangent of the PMMA, were obtained by means of continuous scanning of the associated conductance spectra. This approach yielded the $T_{\mathrm{g}}$ of the PMMA microwire.

QTFs were purchased from the Beijing ChenXing Radio Elements and Material Factory, with a resonance frequency of $\sim 32 \mathrm{kHz}$ and a $\mathrm{Q}$ factor of $>10000$ in a sealed vacuum metallic package and 8000 to 10000 in air after removal of the metallic housing. The QTF prongs have dimensions of $3.65 \mathrm{~mm}, 0.57 \mathrm{~mm}, 0.33 \mathrm{~mm}$, and $0.29 \mathrm{~mm}$ in length, width, thickness, and gap spacing, respectively. The effective spring constant of the QTF is $\sim 20 \mathrm{kN} / \mathrm{m}$. The PMMA powder was purchased from Aldrich (St. Louis, MO). The datasheet from the manufacturer indicates that the weight-average molecular weight was $15000 \mathrm{~g} / \mathrm{mol}$ as measured by gel permeation chromatography (GPC), and the glass transition and autoignition temperatures were $105^{\circ} \mathrm{C}$ and $304^{\circ} \mathrm{C}$, respectively. Initially, the PMMA powder was heated by a resistive heater to $270^{\circ} \mathrm{C}$ above the melting point for $5 \mathrm{~min}$. Then, a sharp tungsten tip with a diameter of $\sim 6 \mu \mathrm{m}$ was used to pull a PMMA wire from the melt. The PMMA microwire was solidified in air and transferred onto a QTF using a threedimensional translation stage. A small amount of epoxy resin adhesive was used to glue the PMMA microwire across the QTF prongs, a process which was monitored by means of an optical microscope. The diameter of the microwire across the QTF prongs in Fig. 1 was measured to be $\sim 10 \mu \mathrm{m}$.

The schematic of the experimental setup is depicted in Fig. 2. The PMMA MM-QTF was installed in a triangular prism-shaped heating cell made of stainless steel. Three sides of the cell were simultaneously heated by three individual resistive heaters. A cylindrical PT100 temperature sensor with a sensitivity of $0.1^{\circ} \mathrm{C}$ was placed close to the MM-QTF to monitor the temperature variations in the cell. The temperature can be controlled from room temperature to $160^{\circ} \mathrm{C}$, at

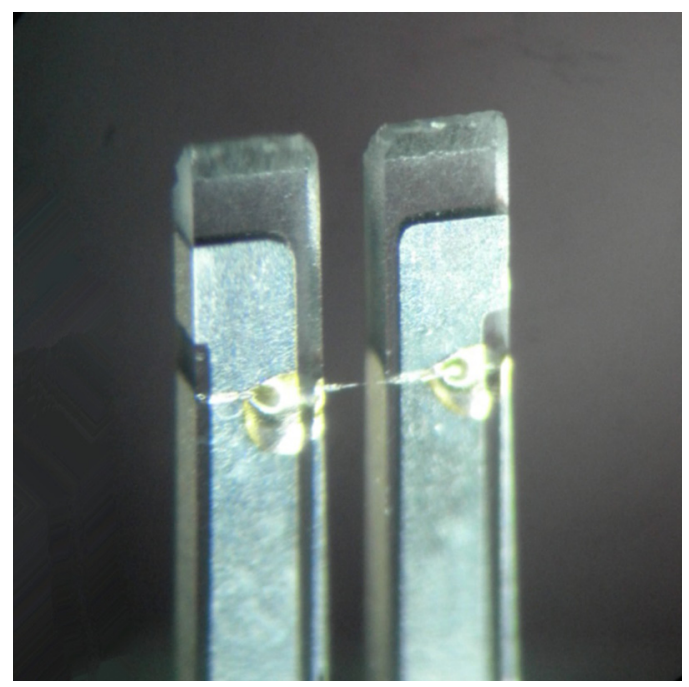

FIG. 1. Photograph of a PMMA microwire modified QTF taken with an optical microscope.

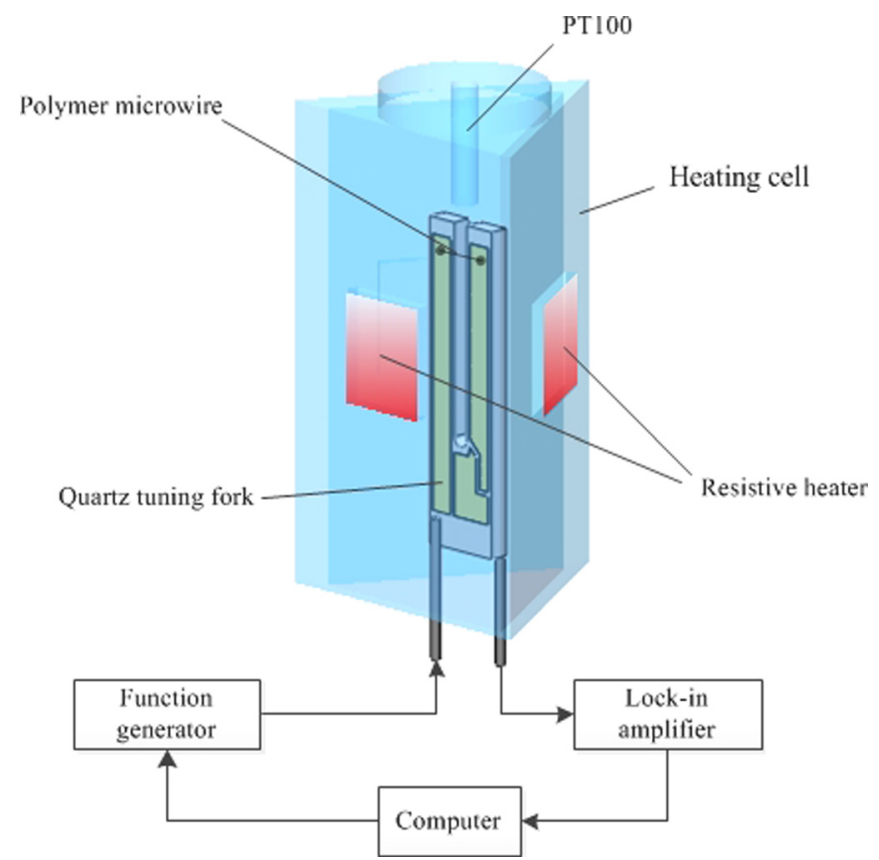

FIG. 2. Schematic of the experimental setup.

a rate of $1{ }^{\circ} \mathrm{C} / \mathrm{min}$ limited by the resistive heaters. The heating and cooling cycles were repeated several times to relieve the stress developed during the PMMA microwire pulling process. The PMMA microwire was subsequently annealed at $135^{\circ} \mathrm{C}$ for $>4 \mathrm{~h}$ before starting the measurements.

A function generator (Agilent 33210 A) was used to generate a sinusoidal wave with a peak-to-peak amplitude of $520 \mathrm{mV}$ for frequency scanning through the resonance frequency of the MM-QTF. The MM-QTF output signal was demodulated by means of a lock-in amplifier (Stanford Research System model SR830) at the $1 f$ mode, with a time constant of $1 \mathrm{~s}$ and a slope of $12 \mathrm{~dB} /$ octave, and then recorded by means of a data acquisition card (DAQ) installed in a computer. The setting parameters of the lock-in amplifier resulted in an electrical detection bandwidth of $0.125 \mathrm{~Hz}$. The output rate of the conductance spectra is 1 spectrum/min, which are limited by the sensor detection bandwidth and the performance of the heaters. The resonance frequency and Q factor of the MM-QTF were calculated from a Lorenz curve fitting to the square of the amplitude associated with the conductance spectra. ${ }^{30}$ All the measurements were performed automatically by means of a self-writing routine based on Labview.

The contrast of the conductance spectra measured at room temperature $\left(26^{\circ} \mathrm{C}\right)$ of the QTF before and after modification by the PMMA microwire is shown in Fig. 3. Compared with a bare QTF, the resonance frequency peak of the PMMA MM-QTF increased from $32760 \mathrm{~Hz}$ to $33687 \mathrm{~Hz}$, yielding a frequency shift of $\Delta f=927 \mathrm{~Hz}$. The resonance frequency $f$ of the QTF can be expressed as

$$
f_{Q T F}=\frac{1}{2 \pi} \times \sqrt{\frac{k_{Q T F}}{m_{Q T F}}}, k_{Q T F}=E_{Q T F} \frac{w t^{3}}{4 l^{3}},
$$

where the $k_{Q T F}, m_{Q T F}$, and $E_{Q T F}$ are the spring constant, effective mass, and Young's modulus of the QTF prongs, and $t, l$, and $w$ represent the thickness, length, and width of the 


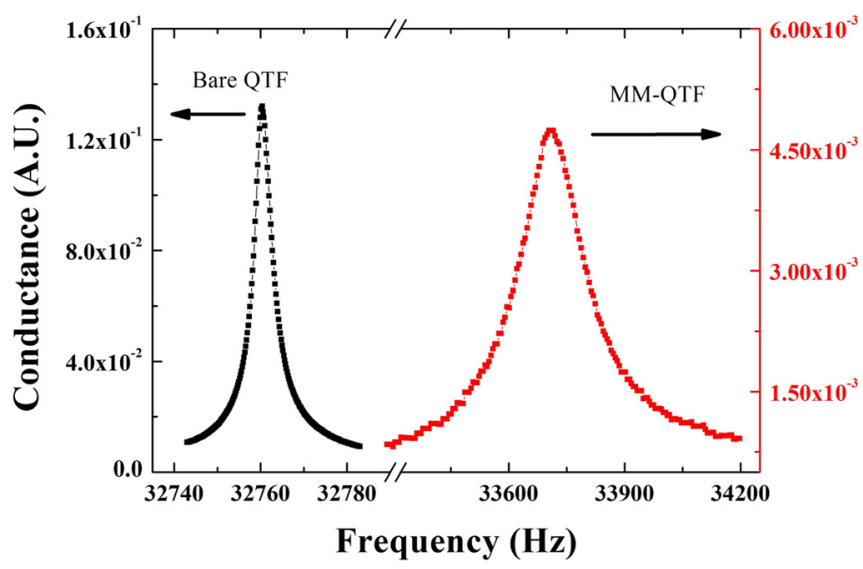

FIG. 3. Conductance spectra of a QTF before (black) and after (red) modification by the PMMA microwire.

QTF prongs, respectively. If we perform a first order partial derivative and take into account that the mass of the polymer microwire is a few nanograms, i.e., $\mathrm{d} m_{Q T F} \ll m_{Q T F}$, the resonance frequency shift, after modification by the PMMA microwire, can be expressed as

$$
\mathrm{d} f_{Q T F}=\frac{f_{Q T F}}{2} \frac{\mathrm{d} k_{Q T F}}{k_{Q T F}} .
$$

The resonance frequency shift can be attributed to the $\mathrm{d} k_{Q T F}$ value, which results from the polymer microwire spring constant $k_{\text {wire }}$, due to the fact that the QTF and the microwire were connected in parallel. Therefore, the effective spring constant $k_{\text {eff }}$ of the PMMA MM-QTF can be expressed as

$$
k_{e f f}=k_{Q T F}+k_{\text {wire }}=E_{Q T F}\left(1+\frac{2 \mathrm{~d} f_{Q T F}}{f_{Q T F}}\right) \frac{w t^{3}}{4 l^{3}}=E_{e f f} \frac{w t^{3}}{4 l^{3}} .
$$

After the $k_{Q T F}$ value in Equation (1) is substituted by $k_{e f f}$, the effective Young's modulus of the PMMA MM-QTF, $E_{e f f}=E_{Q T F}\left(1+\frac{2 \mathrm{~d} f_{Q T F}}{f_{Q T F}}\right)$, is proportional to the square of the resonance frequency.

Figure 4 shows that the resonance frequency change of the PMMA MM-QTF with temperature varies from room temperature $\left(26^{\circ} \mathrm{C}\right)$ to $160{ }^{\circ} \mathrm{C}$. The resonance frequency decreased monotonically from $33687 \mathrm{~Hz}$ at $26^{\circ} \mathrm{C}$ to 32714 at $160^{\circ} \mathrm{C}$, with a $\Delta f=973 \mathrm{~Hz}$. The curve was fitted using piecewise-linear functions, from $26^{\circ} \mathrm{C}$ to $70{ }^{\circ} \mathrm{C}$ and $70^{\circ} \mathrm{C}$ to $160^{\circ} \mathrm{C}$. The slopes of the lines were -4.84 and -8.42 , respectively, indicating that the resonance frequency before $70^{\circ} \mathrm{C}$ decreased more gradually than after $70^{\circ} \mathrm{C}$. However, no clear boundary was found between the glass transition region and the rubbery plateau region. The inset of Fig. 4 shows the resonance frequency change of the bare QTF without polymer wire as a function of temperature. The resonance frequency is almost constant, which indicates that the large $\Delta f$ value is caused by the modulus change of the polymer microwire.

Figure 5 depicts the variations of the $\mathrm{Q}$ factor for the PMMA MM-QTF with temperature. Due to damping of the polymer wire, the Q factor of the QTF decreases from 9773

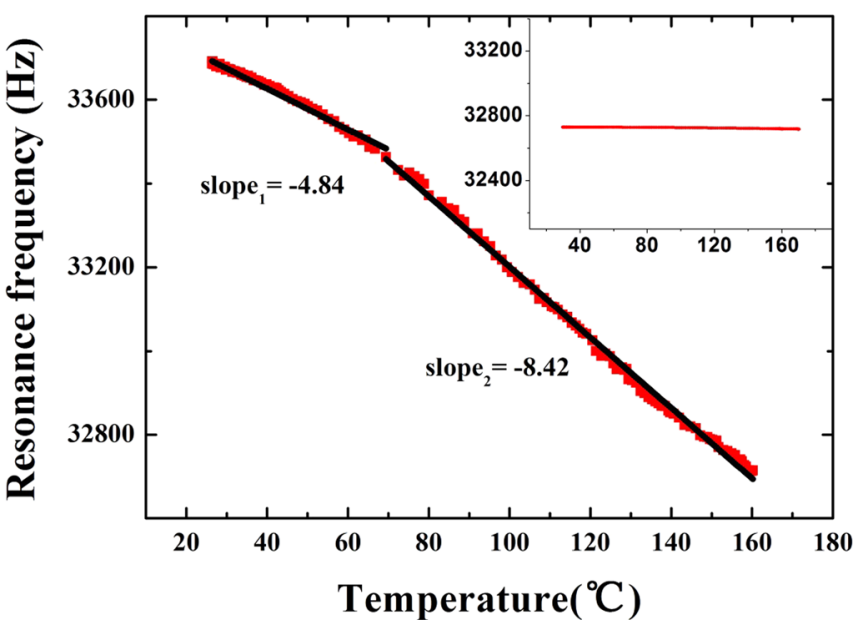

FIG. 4. Resonance frequency variations of a PMMA microwire modified QTF as a function of temperature. The figure inset shows the resonance frequency variations of an unmodified QTF.

to 220 at room temperature. The $\mathrm{Q}$ factor of a resonator represents the ratio of energy stored to energy dissipated per cycle. Hence, a low Q factor means a high energy dissipation rate in the polymer wire. The minimum $\mathrm{Q}$ factor located at $\sim 100^{\circ} \mathrm{C}$ indicates that the maximum energy dissipation rate occurred at this temperature. The inset of Fig. 5 shows a control experiment of the Q factor change for the bare QTF as a function of temperature. In contrast with a change rate of $\sim 9 \%$ and a monotonic decreasing trend of $\mathrm{Q}$ factor for the bare QTF, a change rate of $\sim 50 \%$ and a valley for the PMMA MM-QTF indicate that the energy storage/dissipation of the polymer wire dominates the $\mathrm{Q}$ factor change. In the dynamic mechanics of polymers, the loss tangent $\tan \delta$ is defined as the ratio of the loss modulus $E^{\prime \prime}$ to the storage modulus $E^{\prime}$, i.e., $\tan \delta=\frac{E^{\prime \prime}}{E^{\prime}} 5$. Since the effective storage modulus $E^{\prime}$ is proportional to the square of resonance frequency $f$, the inverse of $\mathbf{Q}$ factor is related to the loss tangent $\tan \delta,{ }^{2}$ and hence the loss modulus $E^{\prime \prime}$ can be expressed as

$$
E^{\prime \prime} \propto \frac{f^{2}}{\mathrm{Q}} .
$$

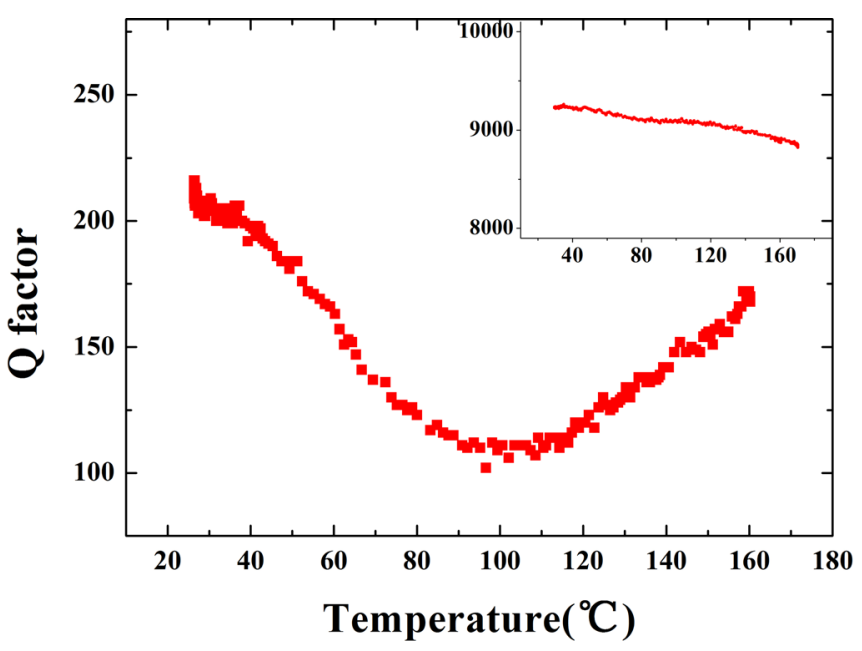

FIG. 5. Q factor variations of a PMMA microwire modified QTF as a function of temperature. The inset shows the $\mathrm{Q}$ factor variations of an unmodified QTF. 


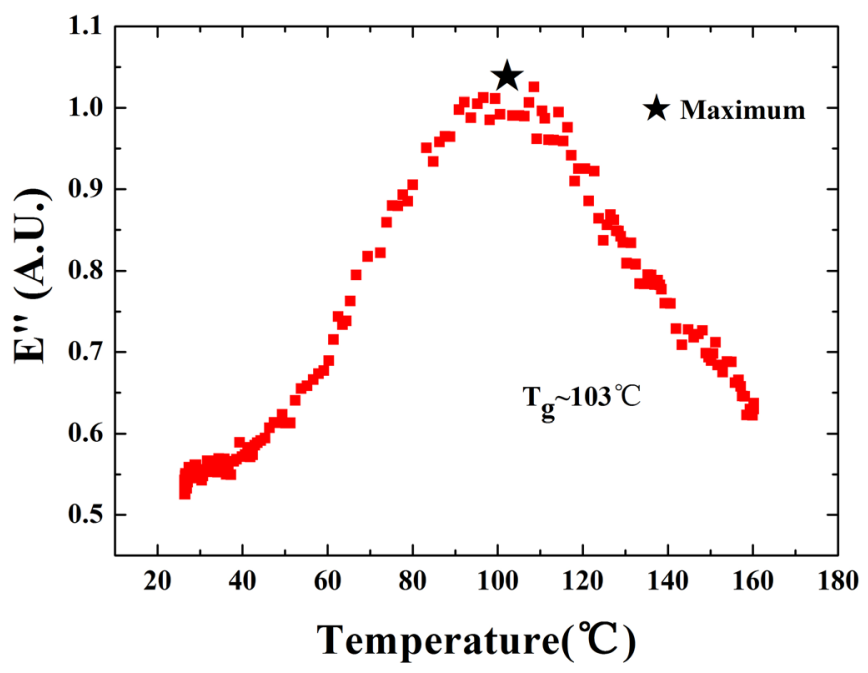

FIG. 6. Loss modulus variations of a PMMA microwire modified QTF as a function of temperature.

The temperature corresponding to the maximum $E^{\prime \prime}$ is usually used as the definition of the glass transition temperature $T_{g}{ }^{10}$ Fig. 6 shows that the $T_{\mathrm{g}}$ of the PMMA determined by the maximum $E^{\prime \prime}$ is $103^{\circ} \mathrm{C}$, which is consistent with the $T_{g} \sim 105^{\circ} \mathrm{C}$ specified by the polymer manufacturer, as measured by the DSC method. However, the DSC technique is a "slow" method with a temperature change rate of $\sim 5 \mathrm{~K} / \mathrm{min}$, compared with the QECS working at $\sim 33 \mathrm{kHz}$. According to the Williams-Landel-Ferry (WLF) equation, a large temperature shift between DSC and QECS methods is expected. The discrepancy may result from the external tension caused by the QTF prongs or from a change of the PMMA feature in a nanogram scale.

Resonance frequency variations of the PMMA MM-QTF showed no difference in the heating and cooling process, as shown in the Fig. 7(a), while a hysteresis effect between $20^{\circ} \mathrm{C}$ and $40^{\circ} \mathrm{C}$ was indicated in the $\mathrm{Q}$ factor variations in Fig. 7(b), due to the physical aging of PMMA. Fig. 7(c) shows that the $T_{\mathrm{g}}$ determined by the maximum $E^{\prime \prime}$ in the heating and cooling process are identical, indicating that the QECS based measurement is reversible. Repetitive measurements were performed to verify the repeatability of the QECS based measurements. As shown in Fig. 7(d), the $E^{\prime \prime}$ variations during the physical aging of three days agreed with each other, indicating that the QECS based nanomechanical analysis is reproducible.

We estimated an error of $\pm 2{ }^{\circ} \mathrm{C}$ for the $T_{\mathrm{g}}$ measurement. This error mainly results from the uncertainty in the actual temperature of the microwires. The maximum uncertainty of the PT-100 temperature sensor within the temperature range used is $\pm 0.1^{\circ} \mathrm{C}$. Considering the finite heat conductivity and radiation loss, the difference in the measured temperature and the actual temperature on the PMMA microwire may reach $\pm 1{ }^{\circ} \mathrm{C}$. In addition, the broad maximum area of the $E^{\prime \prime}$ vs temperature curve generated an error of $\pm 1^{\circ} \mathrm{C}$.

In summary, a $T_{\mathrm{g}}$ determination of PMMA was performed by an analytical tool, QECS. QECS facilitates measurements of a reduced sample size of a few nanograms by $\sim 6$ order of magnitude compared with $5-10 \mathrm{mg}$ in the DSC method. Such a small thermal mass reduces the thermal equilibrium time, which makes QECS a fast and highly sensitive tool for measuring phase transitions of polymer samples at the nanoscale. In addition to the resonance frequency and $\mathrm{Q}$ factor, the equivalent capacitance, inductance, and resistance of a MM-QTF can also be obtained from the information-rich conductance spectra, which are useful to probe the nanomechanical properties of polymers at the nanoscale. Currently, the acquisition time for the resonance frequency and $\mathrm{Q}$ factor requires $\sim 1 \mathrm{~min}$. This can be further shortened by replacing the heater with a faster Peltier element or designing a better electro-magnetic interference (EMI) shielding around the MM-QTF, which would allow increasing the detection bandwidth. Future work will include a dynamic mechanics investigation of polymer nanowires of various diameters. Furthermore, the QTF can be modified by several microwires

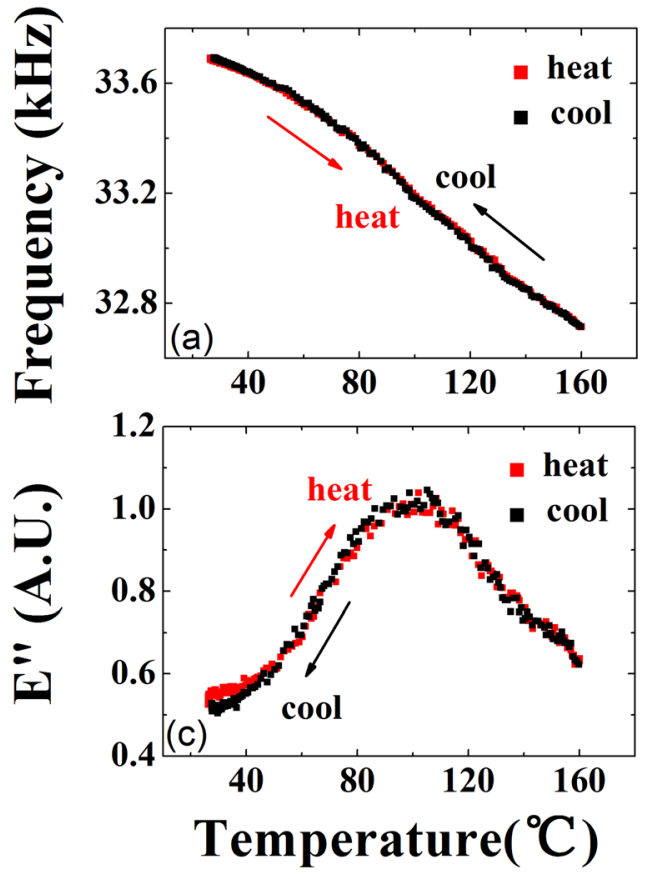

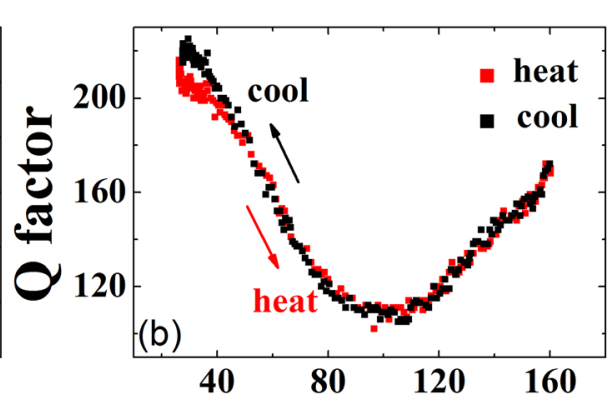

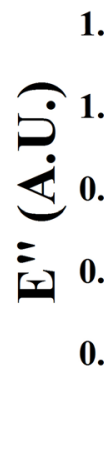

FIG. 7. (a)-(c) Correspond to resonance frequency, Q factors, and loss modulus variations of a PMMA microwire modified QTF as a function of temperature in the heating and cooling process (d) loss modulus variations as a function of time. 
or nanowires of multi-polymers or blends bridged across its prongs. The anticipated $Q$ factor variations as a function of temperature from conductance spectra will show several valleys/dips located at different temperatures. Hence, the glass transition temperatures for these polymers or blends can be measured simultaneously. In addition, QECS can be used to accurately probe the melting point of nanomaterials since there will be a steep jump of the $\mathrm{Q}$ factor when the micro- or nanowires, bridged across the QTF prongs, suddenly break.

Lei Dong acknowledges support by the 973 program (Grant No. 2012CB921603), National Natural Science Foundation of China (Grant Nos. 61575113, 61275213, 61108030, 61475093, 61127017, 61178009, 61378047, and 61205216), the National Key Technology R\&D Program (No. 2013BAC14B01), the Shanxi Natural Science Foundation (No. 2013021004-1), and the Shanxi Scholarship Council of China (2013-011, 2013-01). Frank K. Tittel acknowledges support by the National Science Foundation (NSF) ERC MIRTHE award and the Robert Welch Foundation (Grant No. C-0586).

${ }^{1}$ K. Niedzwiedz, A. Wischnewski, M. Monkenbusch, D. Richter, A. C. Genix, A. Arbe, J. Colmenero, M. Strauch, and E. Straube, Phys. Rev. Lett. 98, 168301 (2007).

${ }^{2}$ N. Jung and S. Jeon, Macromolecules 41, 9819 (2008).

${ }^{3}$ D. Wöll, E. Braeken, A. Deres, F. C. De Schryver, H. Uji-I, and J. Hofkens, Chem. Soc. Rev. 38, 313 (2009).

${ }^{4}$ S. Bose, S. Schmid, T. Larsen, S. S. Keller, P. Sommer-Larsen, A. Boisen, and K. Almdal, ACS Macro Lett. 3, 55 (2014).

${ }^{5}$ L. H. Sperling, Introduction to Physical Polymer Science (Wiley, New York, 2006).

${ }^{6}$ M. Yun, C. Yim, N. Jung, S. Kim, T. Thundat, and S. Jeon, Macromolecules 44, 9661 (2011).

${ }^{7}$ C. Ayela, S. M. Heinrich, F. Josse, and I. Dufour, J. Microelectromech. Syst. 20, 788 (2011).

${ }^{8}$ M. Barbic, L. Eliason, and J. Ranshaw, Sens. Actuators, A 136, 564 (2007).
${ }^{9}$ U. W. Paetzold, S. Lehnen, K. Bittkau, U. Rau, and R. Carius, Nano Lett. 14, 6599 (2014)

${ }^{10}$ F. J. Giessibl, Appl. Phys. Lett. 73, 3956 (1998).

${ }^{11}$ J. P. Waclawek, R. Lewicki, H. Moser, M. Brandstetter, F. K. Tittel, and B. Lendl, Appl. Phys. B 117, 113 (2014).

${ }^{12}$ S. Borri, P. Patimisco, I. Galli, D. Mazzotti, G. Giusfredi, N. Akikusa, M. Yamanishi, G. Scamarcio, P. De Natale, and V. Spagnolo, Appl. Phys. Lett. 104, 091114 (2014).

${ }^{13}$ Y. F. Ma, R. Lewicki, M. Razeghi, and F. K. Tittel, Opt. Express 21, 1008 (2013).

${ }^{14}$ H. M. Yi, R. Maamary, X. M. Gao, M. W. Sigrist, E. Fertein, and W. D. Chen, Appl. Phys. Lett. 106, 101109 (2015).

${ }^{15}$ L. Dong, V. Spagnolo, R. Lewicki, and F. K. Tittel, Opt. Express 19, 24037 (2011).

${ }^{16}$ L. Dong, R. Lewicki, K. Liu, P. R. Buerki, M. J. Weida, and F. K. Tittel, Appl. Phys. B 107, 275 (2012).

${ }^{17}$ H. P. Wu, A. Sampaolo, L. Dong, P. Patimisco, X. L. Liu, H. D. Zheng, X. K. Yin, W. G. Ma, L. Zhang, W. B. Yin, V. Spagnolo, S. T. Jia, and F. K. Tittel, Appl. Phys. Lett. 107, 111104 (2015).

${ }^{18}$ H. P. Wu, L. Dong, H. D. Zheng, X. L. Liu, X. K. Yin, W. G. Ma, L. Zhang, W. Yin, S. T. Jia, and F. K. Tittel, Sens. Actuators, B 221, 666 (2015).

${ }^{19}$ H. D. Zheng, L. Dong, X. K. Yin, X. L. Liu, H. P. Wu, L. Zhang, W. G. Ma, W. B. Yin, and S. Jia, Sens. Actuators, B 208, 173 (2015).

${ }^{20}$ A. Niguès, A. Siria, P. Vincent, P. Poncharal, and L. Bocquet, Nat. Mater. 13, 688 (2014).

${ }^{21}$ S. Boussaad and N. J. Tao, Nano Lett. 3, 1173 (2003).

${ }^{22}$ M. Ren, E. S. Forzani, and N. J. Tao, Anal. Chem. 77, 2700 (2005).

${ }^{23}$ S. Lee, M. Yun, and S. Jeon, Appl. Phys. Lett. 104, 053506 (2014).

${ }^{24}$ M. Meincken, L. J. Balk, and R. D. Sanderson, Macromol. Mater. Eng. 286, 412 (2001).

${ }^{25}$ G. G. Bumbu, M. Wolkenhauer, G. Kircher, J. S. Gutmann, and R. Berger, Langmuir 23, 2203 (2007).

${ }^{26}$ Y. Tian, P. Navarro, and M. Orrit, Phys. Rev. Lett. 113, 135505 (2014).

${ }^{27}$ M. C. Phillips, T. L. Myers, M. D. Wojcik, and B. D. Cannon, Opt. Lett. 32, 1177 (2007).

${ }^{28}$ L. Dong, H. P. Wu, H. D. Zheng, Y. Y. Liu, X. L. Liu, W. Jiang, L. Zhang, W. G. Ma, W. Ren, W. B. Yin, S. T. Jia, and F. K. Tittel, Opt. Lett. 39, 2479 (2014)

${ }^{29}$ D. Weidmann, A. A. Kosterev, F. K. Tittel, N. Ryan, and D. McDonald, Opt. Lett. 29, 1837 (2004).

${ }^{30}$ A. A. Kosterev, F. K. Tittel, D. V. Serebryakov, A. L. Malinovsky, and I. V. Morozov, Rev. Sci. Instrum. 76, 043105 (2005). 